\title{
A Constituent Quark-Meson Model for Heavy-Meson Processes
}

\author{
A. Deandrea \\ Centre de Physique Théorique*, CNRS Luminy, Case 907, F-13288 Marseille Cedex 9, France \\ N. Di Bartolomeo \\ Sissa, via Beirut 2-4, I-34014 Trieste, Italy \\ R. Gatto \\ Département de Physique Théorique, Université de Genève, 24 quai E.-Ansermet, CH-1211 Genève 4, Switzerland \\ G. Nardulli and A. D. Polosa \\ Dipartimento di Fisica, Università di Bari and INFN Bari, via Amendola 173, I-70126 Bari, Italy
}

(February 1998)

\begin{abstract}
We describe the effective heavy meson lagrangian for S- and P-wave heavy-light mesons in terms of a model based on meson-quark interactions, where mesonic transition amplitudes are represented by diagrams with heavy mesons attached to loops containing heavy and light constituent quarks. The model is relativistic and incorporates the heavy quark symmetries. The universal form factors of the heavy meson transition amplitudes are calculated together with their slopes and compared to existing data and limits. As further applications of the model, strong and radiative decays of $D^{*}$ and $B^{*}$ are considered. The agreement with data is surprisingly good and shows that the model offers a viable alternative to effective meson lagrangians which require a larger number of input parameters.
\end{abstract}

\section{INTRODUCTION}

An effective theory for heavy mesons, implementing the heavy quark symmetries, has been very successful at the phenomenological level (for reviews see [1] and references therein). Predictions are easily obtained once the unknown effective couplings are fixed from experimental data. Moreover such an effective approach can be combined with chiral symmetry for light mesons, thus giving a simple framework for implementing the known approximate symmetries of quantum chromodynamics (QCD) [2]. The disadvantage of such an approach is the number of free parameters, which grows very rapidly if one tries to improve the calculations beyond leading order.

In order to go beyond the symmetry approach, one should be able to derive the free couplings at the meson lagrangian level from a more fundamental theory, for example directly from QCD. This is clearly a difficult task. In the long run the definitive answer will come from systematic first principle calculations, such as in lattice QCD, but at present heavy meson physical quantities such as spectra and form factors are still subject to extrapolations, even if recent improvements are impressive [3]. Moreover an alternative and intuitive way for interpolating between QCD and an effective theory would be interesting in itself, allowing us to understand better the underlying physics. Obviously there is a price to pay for any simplification that may allow to calculate the parameters of an effective heavy meson theory without solving the nonperturbative QCD problem. Our point of view here will be to consider a quark-meson lagrangian where transition amplitudes are represented by diagrams with heavy mesons attached to loops containing heavy and light constituent quarks. It should be kept in mind that what we study here is only a model and not full QCD. However one can hope to describe the essential part of the QCD behavior in some energy range and extract useful information from it.

Since the model used in the present paper is based on an effective constituent quark-meson lagrangian containing both light and heavy degrees of freedom it is constrained by the known symmetries of QCD in the limit $m_{Q} \rightarrow \infty$. A similar model, for light quarks only, was pioneered, in the context of chirally symmetric effective theories, several years ago in [4]. A related approach is the one based on the extended Nambu Jona-Lasinio (NJL) models [5], whose generalization to include both heavy and light quarks has been studied in [6]. In this approach path integral

*Unité Propre de Recherche 7061 
bosonization is used, which replaces the effective 4-quark interactions by Yukawa-type couplings of heavy and light quarks with heavy meson fields. One may think of going even further and try to bosonize directly QCD, but a number of approximations are needed and one usually ends up with a non-local lagrangian.

In the following we are interested in the description of the heavy meson interactions for the doublets with spin/parity $J^{P}=\left(1^{+}, 0^{+}\right),\left(1^{-}, 0^{-}\right),\left(2^{+}, 1^{+}\right)$, i.e. S- and P-wave heavy-light mesons. These states are the object of experimental search [7]. Our aim is therefore to extend the approach of [ [ 1 and [6] to the description of the three spin-parity doublets mentioned above. We will consider a lagrangian at the meson-quark level, fixing the free parameters from data. This will allow us to deduce from a small number of parameters the heavy meson couplings and form factors, with a considerable reduction in the number of free parameters with respect to the effective lagrangian written in terms of meson fields only.

The plan of the paper is as follows. In section 2 we give an outline of the model. In Section 3 we compute some fundamental parameters of the model, such as mass splittings and renormalization constants. The model is used in Section 4 to obtain predictions for semileptonic $B$ decays into negative parity and positive parity charmed resonances; in particular we compute the relevant universal form factors that describe such decays in the $m_{Q} \rightarrow \infty$ limit and discuss the Bjorken sum rule [8]. In section 5 we compute strong and radiative $D^{*}$ and $B^{*}$ decays. Finally in Section 6 we draw our conclusions.

\section{THE MODEL}

To begin with we briefly review the description of the heavy quark field in the heavy quark effective theory (HQET) [1]. The heavy quark is defined as follows: we indicate by $v_{\mu}$ the velocity of the hadron containing the heavy quark $Q$. This is almost on shell and its momentum $p_{Q}$ can be written, introducing a residual momentum $k$, as

$$
p_{Q}=m_{Q} v+k
$$

One extracts the dominant part $m_{Q} v$ of the heavy quark momentum by defining the new field $Q_{v}$

$$
Q_{v}(x)=\exp \left(i m_{Q} v x\right) Q(x)=h_{v}(x)+H_{v}(x) .
$$

The field $h_{v}$ is the large component field, satisfying the constraint $\not h_{v}=h_{v}$. If the quark $Q$ is exactly on shell, it is the only term present in the previous equation. $H_{v}$, the small component field, is of the order $1 / m_{Q}$ and satisfies $\not H_{v}=-H_{v}$. The effective lagrangian can be derived by integrating out the heavy fields in the QCD generating functional [9], however here we only show the effect of the $m_{Q} \rightarrow \infty$ limit on the Feynman rules relevant to our calculation [10]. The heavy quark propagator in the large $m_{Q}$ limit is:

$$
\frac{i}{\not p-m_{Q}} \simeq \frac{i}{v \cdot k} \frac{1+\not p}{2} \text {. }
$$

The $(1+\not p) / 2$ projection operator can always be moved close to a spinor $h_{v}$ satisfying $\not h h_{v}=h_{v}$ since $\not b$ commutes with the heavy quark-gluon vertex.

In our model the heavy quark propagator appears in the loops always together with light quark propagators as the heavy mesons we consider are made up of constituent heavy quark and light antiquark. The light-quark momentum $k$ may be taken equal to the integrated loop momentum and the heavy-quark propagator in the heavy quark limit becomes:

$$
\frac{i}{v \cdot k+\Delta}
$$

where $\Delta$ is the difference between the quark mass and the common mass of the heavy meson doublet.

\section{A. Heavy meson fields}

In order to implement the heavy quark symmetries in the spectrum of physical states the wave function of a heavy meson has to be independent of the heavy quark flavor and spin. It can be characterized by the total angular momentum $s_{\ell}$ of the light degrees of freedom. To each value of $s_{\ell}$ corresponds a degenerate doublet of states with 
angular momentum $J=s_{\ell} \pm 1 / 2$. The mesons $P$ and $P^{*}$ form the spin-symmetry doublet corresponding to $s_{\ell}=1 / 2$ (for charm for instance, they correspond to $D$ and $D^{*}$ ).

The negative parity spin doublet $\left(P, P^{*}\right)$ can be represented by a $4 \times 4$ Dirac matrix $H$, with one spinor index for the heavy quark and the other for the light degrees of freedom. These wave functions transform under a Lorentz transformation $\Lambda$ as

$$
H \rightarrow D(\Lambda) H D(\Lambda)^{-1}
$$

where $D(\Lambda)$ is the usual $4 \times 4$ representation of the Lorentz group. Under a heavy quark spin transformation $S$, $H \rightarrow S H \quad$, where $S$ satisfies $[\not, S]=0$ to preserve the constraint $\not H H=H$.

An explicit matrix representation is:

$$
\begin{aligned}
H & =\frac{(1+\not b)}{2}\left[P_{\mu}^{*} \gamma^{\mu}-P \gamma_{5}\right] \\
\bar{H} & =\gamma_{0} H^{\dagger} \gamma_{0} .
\end{aligned}
$$

Here $v$ is the heavy meson velocity, $v^{\mu} P_{a \mu}^{*}=0$ and $M_{H}=M_{P}=M_{P^{*}}$. Moreover $\not b H=-H \not b=H, \bar{H} \not \varnothing=-\not \bar{H}=\bar{H}$ and $P^{* \mu}$ and $P$ are annihilation operators normalized as follows:

$$
\begin{aligned}
\left\langle 0|P| Q \bar{q}\left(0^{-}\right)\right\rangle & =\sqrt{M_{H}} \\
\left\langle 0\left|P^{* \mu}\right| Q \bar{q}\left(1^{-}\right)\right\rangle & =\epsilon^{\mu} \sqrt{M_{H}} .
\end{aligned}
$$

The formalism for higher spin states is given in [11]. We will consider only the extension to $P$-waves of the system $Q \bar{q}$. The heavy quark effective theory predicts two distinct multiplets, one containing a $0^{+}$and a $1^{+}$degenerate state, and the other one $1^{+}$and a $2^{+}$state. In matrix notations, analogous to the ones used for the negative parity states, they are described by

$$
S=\frac{1+\not p}{2}\left[P_{1 \mu}^{* \prime} \gamma^{\mu} \gamma_{5}-P_{0}\right]
$$

and

$$
T^{\mu}=\frac{1+\not}{2}\left[P_{2}^{* \mu \nu} \gamma_{\nu}-\sqrt{\frac{3}{2}} P_{1 \nu}^{*} \gamma_{5}\left(g^{\mu \nu}-\frac{1}{3} \gamma^{\nu}\left(\gamma^{\mu}-v^{\mu}\right)\right)\right] .
$$

These two multiplets have $s_{\ell}=1 / 2$ and $s_{\ell}=3 / 2$ respectively, where $s_{\ell}$ is conserved together with the spin $s_{Q}$ in the infinite quark mass limit because $\vec{J}=\vec{s}_{\ell}+\vec{s}_{Q}$.

\section{B. Interaction terms}

The effective quark-meson lagrangian we introduce contains two terms:

$$
\mathcal{L}=\mathcal{L}_{\ell \ell}+\mathcal{L}_{h \ell} .
$$

The first term involves only the light degrees of freedom, i.e. the light quark fields $\chi$ and the pseudo-scalar $S U(3)$ octet of mesons $\pi$. At the lowest order one has:

$$
\begin{aligned}
\mathcal{L}_{\ell \ell} & =\bar{\chi}\left(i D^{\mu} \gamma_{\mu}+g_{A} \mathcal{A}^{\mu} \gamma_{\mu} \gamma_{5}\right) \chi-m \bar{\chi} \chi \\
& +\frac{f_{\pi}^{2}}{8} \partial_{\mu} \Sigma^{\dagger} \partial^{\mu} \Sigma .
\end{aligned}
$$

Here $D_{\mu}=\partial_{\mu}-i \mathcal{V}_{\mu}, \xi=\exp \left(i \pi / f_{\pi}\right), \Sigma=\xi^{2}, f_{\pi}=130 \mathrm{MeV}$ and

$$
\begin{aligned}
\mathcal{V}^{\mu} & =\frac{1}{2}\left(\xi^{\dagger} \partial^{\mu} \xi+\xi \partial^{\mu} \xi^{\dagger}\right) \\
\mathcal{A}^{\mu} & =\frac{i}{2}\left(\xi^{\dagger} \partial^{\mu} \xi-\xi \partial^{\mu} \xi^{\dagger}\right) .
\end{aligned}
$$


The term with $g_{A}$ is the coupling of pions to light quarks; it will not be used in the sequel. It is a free parameter, but in NJL model $g_{A}=1$. Differently from [4] the gluon field is absent in the present description. Apart from the mass term $\mathcal{L}_{\ell \ell}$ is chiral invariant.

We can introduce a quark-meson effective lagrangian involving heavy and light quarks and heavy mesons. At lowest order we have:

$$
\begin{aligned}
\mathcal{L}_{h \ell} & =\bar{Q}_{v} i v \cdot \partial Q_{v}-\left(\bar{\chi}\left(\bar{H}+\bar{S}+i \bar{T}_{\mu} \frac{D^{\mu}}{\Lambda_{\chi}}\right) Q_{v}+\text { h.c. }\right) \\
& +\frac{1}{2 G_{3}} \operatorname{Tr}[\bar{H} H]+\frac{1}{2 G_{3}^{\prime}} \operatorname{Tr}[\bar{S} S]+\frac{1}{2 G_{4}} \operatorname{Tr}\left[\bar{T}_{\mu} T^{\mu}\right]
\end{aligned}
$$

where the meson fields $H, S, T$ have been defined before, $Q_{v}$ is the effective heavy quark field, $G_{3}, G_{3}^{\prime}, G_{4}$ are coupling constants (to allow a comparison with previous work we follow, as far as possible, the notations of [6]) and $\Lambda_{\chi}(=1 \mathrm{GeV})$ has been introduced for dimensional reasons. Lagrangian (15) has heavy spin and flavor symmetry. This lagrangian comprises three terms containing respectively $H, S$ and $T$. From a theoretical point of view, the $G_{3}$ and $G_{3}^{\prime}$ couplings could have been determined by $\Delta_{H}$ and $\Delta_{S}$, the experimental values of the mass differences between the mesons, belonging to the $\mathrm{H}$ and $\mathrm{S}$ multiplets respectively, and their heavy quark constituent masses. Since these data are not all known, we have adopted the dynamical information coming from the NJL-model as proposed in [6] and so we use the following modified version of (15) in which we give the fields $H$ and $S$ the same coupling to the quarks:

$$
\begin{aligned}
\mathcal{L}_{h \ell} & =\bar{Q}_{v} i v \cdot \partial Q_{v}-\left(\bar{\chi}\left(\bar{H}+\bar{S}+i \bar{T}_{\mu} \frac{D^{\mu}}{\Lambda_{\chi}}\right) Q_{v}+\text { h.c. }\right) \\
& +\frac{1}{2 G_{3}} \operatorname{Tr}[(\bar{H}+\bar{S})(H-S)]+\frac{1}{2 G_{4}} \operatorname{Tr}\left[\bar{T}_{\mu} T^{\mu}\right]
\end{aligned}
$$

In doing this we assume that this effective quark meson lagrangian can be justified as a remnant of a four quark interaction of the NJL type by partial bosonization. $H$ and $S$ are degenerate in mass in the light-sector chirally symmetric phase. In the broken phase the mass splitting is calculable and will be computed in the next section in terms of the order parameter $m$, the constituent light quark mass. The part containing the $T$ field can not be extracted from a bosonized NJL contact interaction and requires a new coupling constant $G_{4}$.

An essential ingredient of the nonperturbative behavior of QCD is the suppression of large momentum flows through light-quark lines in the loops. The way this suppression is introduced in the model is crucial and is part of the definition of the model itself. One can for example include factors in the vertices which damp the loop integrals when the lightquark momentum is larger than some scale of the order of $1 \mathrm{GeV}$ 12]. Our model describes the interactions in terms of effective vertices between a light quark, a heavy quark and a heavy meson; we describe the heavy quarks and heavy mesons consistently with HQET, and thus the heavy quark propagator in the loop contains the residual momentum $k$ which arises from the interaction with the light degrees of freedom. It is therefore natural to assume an ultraviolet cut-off on the loop momentum of the order of $\Lambda \simeq 1 \mathrm{GeV}$, even if the heavy quark mass is larger than the cut-off.

Concerning the infrared behavior, the model is not confining and thus its range of validity can not be extended below energies of the order of $\Lambda_{Q C D}$. In practice one introduces an infrared cut-off $\mu$, in order to drop the unknown confinement part of the quark interaction.

We choose a proper time regularization (a different choice is followed in [13]). After continuation to the Euclidean space it reads, for the light quark propagator:

$$
\int d^{4} k_{E} \frac{1}{k_{E}^{2}+m^{2}} \rightarrow \int d^{4} k_{E} \int_{1 / \mu^{2}}^{1 / \Lambda^{2}} d s e^{-s\left(k_{E}^{2}+m^{2}\right)}
$$

where $\mu$ and $\Lambda$ are infrared and ultraviolet cut-offs. This choice of regularization is consistent with the one in the second paper in [6]. In (17) $m$ is the constituent light quark mass, whose non vanishing value implies the mass splitting between the $H$-type and $S$-type multiplets. Its value, as well as the values of the cutoffs $\mu$ and $\Lambda$ are in principle adjustable parameters. On physical grounds, we expect that $\Lambda \simeq 1 \mathrm{GeV}, m \simeq \mu \simeq 10^{2}$ MeV. Given our choice of the cut-off prescription as in [6], we assume for $\Lambda$ the same value used there i.e. $\Lambda=1.25 \mathrm{GeV}$; we observe, however, that the numerical outcome of the subsequent calculations are not strongly dependent on the value of $\Lambda$. The constituent mass $m$ in the NJL models represents the order parameter discriminating between the phases of broken and unbroken chiral symmetry and can be fixed by solving a gap equation, which gives $m$ as a function of the scale mass $\mu$ for given values of the other parameters. In the second paper of ref. [6] the values $m=300 \mathrm{MeV}$ and $\mu=300 \mathrm{MeV}$ are used and we shall assume the same values. As shown there, for smaller values of $\mu, m$ is constant $(=300 \mathrm{MeV})$ while for much larger values of $\mu$, it decreases and in particular it vanishes for $\mu=550 \mathrm{MeV}$. 


\section{RENORMALIZATION CONSTANTS AND MASSES}

For the computation of the constants $G_{3}, G_{4}$ appearing in the quark-meson effective lagrangian of Eq. 15. we consider the meson self energy diagram depicted in Fig.1.

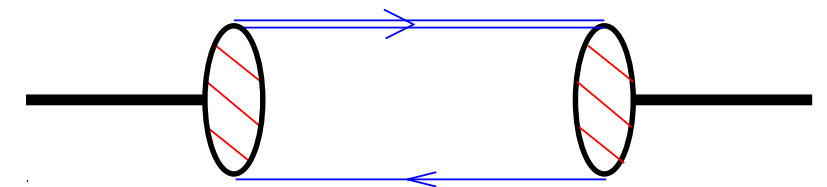

Fig. 1 - Self-energy diagram for the heavy meson field.

For $H$ and $S$ states the evaluation of Fig.1 gives the results ( $k$ is the residual momentum, see eq. (11)):

$$
\operatorname{Tr}\left[-\bar{H} \Pi_{H}(v \cdot k) H\right]=i N_{c} \int \frac{d^{4} \ell}{(2 \pi)^{4}} \frac{\operatorname{Tr}[(\ell-\not k-m) \bar{H} H]}{\left((\ell-k)^{2}-m^{2}+i \epsilon\right)(v \ell+i \epsilon)}
$$

and

$$
\operatorname{Tr}\left[+\bar{S} \Pi_{S}(v \cdot k) S\right]=i N_{c} \int \frac{d^{4} \ell}{(2 \pi)^{4}} \frac{\operatorname{Tr}[(\ell-\not k+m) \bar{S} S]}{\left((\ell-k)^{2}-m^{2}+i \epsilon\right)(v \ell+i \epsilon)}
$$

respectively, and for $T$ states we get

$$
\operatorname{Tr}\left[+\bar{T}_{\mu} \Pi_{T}(v \cdot k) T^{\mu}\right]=\frac{i N_{c}}{\Lambda_{\chi}^{2}} \int \frac{d^{4} \ell}{(2 \pi)^{4}} \frac{\operatorname{Tr}\left[\left(\ell_{\mu}-k_{\mu}\right)(\ell-\not k+m)\left(\ell_{\nu}-k_{\nu}\right) \bar{T}^{\mu} T^{\nu}\right]}{\left((\ell-k)^{2}-m^{2}+i \epsilon\right)(v \ell+i \epsilon)}
$$

We notice that the only difference between $\Pi_{H}$ and $\Pi_{S}$ is in the sign in front of $m$ in the numerators of the integrands. We now expand $(18),(19)$ and 20 ) around $\Delta_{H}, \Delta_{S}$ and $\Delta_{T}$ respectively:

$$
\Pi(v \cdot k) \simeq \Pi(\Delta)+\Pi^{\prime}(\Delta)(v \cdot k-\Delta) .
$$

In this way we obtain the kinetic part of the effective lagrangian for heavy mesons in the usual form [2]

$$
\begin{aligned}
\mathcal{L}_{e f f} & =\operatorname{Tr} \overline{\hat{H}}\left(i v \cdot \partial-\Delta_{H}\right) \hat{H}+\operatorname{Tr} \overline{\hat{S}}\left(i v \cdot \partial-\Delta_{S}\right) \hat{S} \\
& +\operatorname{Tr} \overline{\hat{T}}_{\mu}\left(i v \cdot \partial-\Delta_{T}\right) \hat{T}^{\mu}
\end{aligned}
$$

provided we satisfy the conditions:

$$
\begin{aligned}
& \frac{1}{2 G_{3}}=\Pi_{H}\left(\Delta_{H}\right)=\Pi_{S}\left(\Delta_{S}\right) \\
& \frac{1}{2 G_{4}}=\Pi_{T}\left(\Delta_{T}\right)
\end{aligned}
$$

and renormalize the fields as :

$$
\begin{gathered}
\hat{H}=\frac{H}{\sqrt{Z_{H}}} \\
\hat{S}=\frac{S}{\sqrt{Z_{S}}} \\
\hat{T}=\frac{T}{\sqrt{Z_{T}}} .
\end{gathered}
$$

The renormalization constants are given by:

$$
Z_{j}^{-1}=\Pi^{\prime}\left(\Delta_{j}\right)
$$

Here, prime denotes differentiation and $j=H, S$ and $T$. In the previous equations, $\Delta_{H}, \Delta_{S}, \Delta_{T}$ are the mass differences between the heavy mesons $H, S, T$ and the heavy quark. The expressions of $\Pi_{H}, \Pi_{S}$, and $\Pi_{T}$ are: 


$$
\begin{aligned}
\Pi_{H}\left(\Delta_{H}\right) & =I_{1}+\left(\Delta_{H}+m\right) I_{3}\left(\Delta_{H}\right) \\
\Pi_{S}\left(\Delta_{S}\right) & =I_{1}+\left(\Delta_{S}-m\right) I_{3}\left(\Delta_{S}\right) \\
\Pi_{T}\left(\Delta_{T}\right) & =\frac{1}{\Lambda_{\chi}^{2}}\left[-\frac{I_{1}^{\prime}}{4}+\frac{m+\Delta_{T}}{3}\left[I_{0}\left(\Delta_{T}\right)+\Delta_{T} I_{1}+\left(\Delta_{T}^{2}-m^{2}\right) I_{3}\left(\Delta_{T}\right)\right]\right]
\end{aligned}
$$

while the field renormalization constants are :

$$
\begin{gathered}
Z_{H}^{-1}=\left(\Delta_{H}+m\right) \frac{\partial I_{3}\left(\Delta_{H}\right)}{\partial \Delta_{H}}+I_{3}\left(\Delta_{H}\right) \\
Z_{S}^{-1}=\left(\Delta_{S}-m\right) \frac{\partial I_{3}\left(\Delta_{S}\right)}{\partial \Delta_{S}}+I_{3}\left(\Delta_{S}\right) \\
Z_{T}^{-1}=\frac{1}{3 \Lambda_{\chi}^{2}}\left[\left(\Delta_{T}^{2}-m^{2}\right)\left[\left(m+\Delta_{T}\right) \frac{\partial I_{3}\left(\Delta_{T}\right)}{\partial \Delta_{T}}+I_{3}\left(\Delta_{T}\right)\right]\right. \\
\left.+\left(m+\Delta_{T}\right)\left[\frac{\partial I_{0}\left(\Delta_{T}\right)}{\partial \Delta_{T}}+I_{1}+2 \Delta_{T} I_{3}\left(\Delta_{T}\right)\right]+I_{0}+\Delta_{T} I_{1}\right]
\end{gathered}
$$

The integrals $I_{0}, I_{1}, I_{1}^{\prime}$ and $I_{3}$ are defined in the Appendix. We employ the first equation in (23) to obtain $G_{3}$ from a given value of $\Delta_{H}$ (we use $\Delta_{H}$ in the range $0.3-0.5 \mathrm{GeV}$ ), while the second equation in (23) can be used to determine $\Delta_{S}$.

For $\Delta_{T}$ we can use experimental information. Let us call $M_{H}$ and $M_{T}$ the spin-averaged $H$-multiplet and $T$-multiplet masses. We can write $M_{H}=m_{Q}+\Delta_{H}+\Delta_{H}^{\prime} / m_{Q}$ and $M_{T}=m_{Q}+\Delta_{T}+\Delta_{T}^{\prime} / m_{Q}$. For charm, the positive parity experimental masses and widths are as follows: for $D_{2}^{*}$ we have $m_{D_{2}^{*}(2460)^{0}}=2458.9 \pm 2.0 \mathrm{MeV}, \Gamma_{D_{2}^{*}(2460)^{0}}=23 \pm 5$ $\mathrm{MeV}$ and $m_{D_{2}^{*}(2460)^{ \pm}}=2459 \pm 4 \mathrm{MeV}, \Gamma_{D_{2}^{*}(2460)^{ \pm}}=25_{-7}^{+8} \mathrm{MeV}$. These particle are identified with the state $2^{+}$ belonging to the $T \frac{3}{2}^{+}$multiplet. As for the $D_{1}^{*}(2420)$ state, experimentally we have $m_{D_{1}^{*}(2420)^{0}}=2422.2 \pm 1.8 \mathrm{MeV}$, $\Gamma_{D_{1}^{*}(2420)^{0}}=18.9_{-3.5}^{+4.6} \mathrm{MeV}$; this particle can be identified with the state $1^{+}$belonging to the $T$ multiplet, neglecting a possible small mixing between the two $1^{+}$states belonging to the $S$ and $T$ multiplets [2]. We observe that the narrowness of the states $D_{2}^{*}$ and $D_{1}^{*}$ is due to the fact that their strong decays occur via $D$-waves. From this analysis we get $\Delta_{T}-\Delta_{H}+\left(\Delta_{T}^{\prime}-\Delta_{H}^{\prime}\right) / m_{c} \simeq 470 \mathrm{MeV}$. For beauty, the experimental data on positive parity resonances show a bunch of states with a mass $M_{B^{* *}}=5698 \pm 12 \mathrm{MeV}$ and width $\Gamma=128 \pm 18 \mathrm{MeV}$ [14. If we identify this mass with the mass of the narrow $T$ states, we obtain $\Delta_{T}-\Delta_{H}+\left(\Delta_{T}^{\prime}-\Delta_{H}^{\prime}\right) / m_{b} \simeq 380 \mathrm{MeV}$. For reasonable values of the heavy quark masses, we get

$$
\Delta_{T}-\Delta_{H} \simeq 335 \mathrm{MeV},
$$

which is the value we adopt. The preceding analysis produces the figures in Table I.

\begin{tabular}{lll}
\hline \hline$\Delta_{H}$ & $\Delta_{S}$ & $\Delta_{T}$ \\
\hline 0.3 & 0.545 & 0.635 \\
0.4 & 0.590 & 0.735 \\
0.5 & 0.641 & 0.835 \\
\hline \hline
\end{tabular}

TABLE I. $\Delta$ values (in $\mathrm{GeV}$ )

In Table II we report the computed values of $G_{j}$ and $Z_{j}$ for three values of $\Delta_{H}$.

Let us finally observe that we predict, according to the value of $\Delta_{H}$, a value for the $S$-multiplet mass $m=2165 \pm 50$ $\mathrm{MeV}$; these states, called in the literature $D_{0}, D_{1}^{* \prime}$ have not been observed yet since they are expected to be rather broad (for instance in [15] one predicts $\Gamma\left(D_{0} \rightarrow D^{+} \pi^{-}\right) \simeq 180 \mathrm{MeV}$ and $\left.\Gamma\left(D_{1}^{* \prime} \rightarrow D^{*+} \pi^{-}\right) \simeq 165 \mathrm{MeV}\right)$. Theoretical predictions in the literature are somehow larger $(m \simeq 2350 \mathrm{MeV})$.

\begin{tabular}{llllll}
\hline \hline$\Delta_{H}$ & $1 / G_{3}$ & $Z_{H}$ & $Z_{S}$ & $Z_{T}$ & $1 / G_{4}$ \\
\hline 0.3 & 0.16 & 4.17 & 1.84 & 2.95 & 0.15 \\
0.4 & 0.22 & 2.36 & 1.14 & 1.07 & 0.26 \\
0.5 & 0.345 & 1.142 & 0.63 & 0.27 & 0.66 \\
\hline \hline
\end{tabular}

TABLE II. Renormalization constants and couplings. $\Delta_{H}$ in $\mathrm{GeV} ; G_{3}, G_{4}$ in $\mathrm{GeV}^{-2}, Z_{j}$ in $\mathrm{GeV}^{-1}$. 


\section{SEMILEPTONIC DECAYS AND FORM FACTORS}

To begin with, we compute the leptonic decay constants $\hat{F}$ and $\hat{F}^{+}$that are defined as follows:

$$
\begin{aligned}
\left\langle 0\left|\bar{q} \gamma^{\mu} \gamma_{5} Q\right| H\left(0^{-}, v\right)\right\rangle & =i \sqrt{M_{H}} v^{\mu} \hat{F} \\
\left\langle 0\left|\bar{q} \gamma^{\mu} Q\right| S\left(0^{+}, v\right)\right\rangle & =i \sqrt{M_{S}} v^{\mu} \hat{F}^{+} .
\end{aligned}
$$

It is easy to compute these constants by a loop calculation similar to that considered in the previous section for the self-energy ; one finds

$$
\begin{aligned}
\hat{F} & =\frac{\sqrt{Z_{H}}}{G_{3}} \\
\hat{F}^{+} & =\frac{\sqrt{Z_{S}}}{G_{3}}
\end{aligned}
$$

The numerical results for different values of the parameters can be found in Table III.

\begin{tabular}{lll}
\hline \hline$\Delta_{H}$ & $\hat{F}$ & $\hat{F}^{+}$ \\
\hline 0.3 & 0.33 & 0.22 \\
0.4 & 0.34 & 0.24 \\
0.5 & 0.37 & 0.27 \\
\hline \hline
\end{tabular}

TABLE III. $\hat{F}$ and $\hat{F}^{+}$for various values of $\Delta_{H} . \Delta_{H}$ in $\mathrm{GeV}$, leptonic constants in $\mathrm{GeV}^{3 / 2}$. 
We observe that, neglecting logarithmic corrections, $\hat{F}$ and $\hat{F}^{+}$are related, in the infinite heavy quark mass limit, to the leptonic decay constant $f_{B}$ and $f^{+}$defined by

$$
\begin{aligned}
\left\langle 0\left|\bar{q} \gamma^{\mu} \gamma_{5} b\right| B(p)\right\rangle & =i p^{\mu} f_{B} \\
\left\langle 0\left|\bar{q} \gamma^{\mu} b\right| B_{0}(p)\right\rangle & =i p^{\mu} f^{+}
\end{aligned}
$$

by the relations $f_{B}=\hat{F} / \sqrt{M_{B}}$ and $f^{+}=\hat{F}^{+} / \sqrt{M_{B_{0}}}$. For example, for $\Delta_{H}=400 \mathrm{MeV}$, we obtain from Table III:

$$
\begin{aligned}
& f_{B} \simeq 150 \mathrm{MeV} \\
& f^{+} \simeq 100 \mathrm{MeV} .
\end{aligned}
$$

The numerical values that can be found in the literature agree with our results. For example the QCD sum rule analysis of [16] gives $\hat{F}=0.30 \pm 0.05 \mathrm{GeV}^{3 / 2}$ (without $\alpha_{s}$ corrections) and higher values (around $0.4-0.5$ $\mathrm{GeV}^{3 / 2}$ ) including radiative corrections. As for lattice $\mathrm{QCD}$, in [3] the value summarizing the present status of lattice calculations for $f_{B}$ is $170 \pm 35 \mathrm{MeV}$. As for $F^{+}$, a QCD sum rule analysis 17] gives $F^{+}=0.46 \pm 0.06 \mathrm{GeV}^{3 / 2}$, which is significantly higher than the results reported in Table III.

Let us now consider semileptonic decays. The first analysis to be performed is the study of the Isgur-Wise function $\xi$ which is defined by:

$$
\begin{aligned}
\left\langle D\left(v^{\prime}\right)\left|\bar{c} \gamma_{\mu}\left(1-\gamma_{5}\right) b\right| B(v)\right\rangle & =\sqrt{M_{B} M_{D}} C_{c b} \xi(\omega)\left(v_{\mu}+v_{\mu}^{\prime}\right) \\
\left\langle D^{*}\left(v^{\prime}, \epsilon\right)\left|\bar{c} \gamma_{\mu}\left(1-\gamma_{5}\right) b\right| B(v)\right\rangle & =\sqrt{M_{B} M_{D^{*}}} C_{c b} \xi(\omega)\left[i \epsilon_{\mu \nu \alpha \beta} \epsilon^{* \nu} v^{\prime \alpha} v^{\beta}\right. \\
& \left.-(1+\omega) \epsilon_{\mu}^{*}+\left(\epsilon^{*} \cdot v\right) v_{\mu}^{\prime}\right]
\end{aligned}
$$

where $\omega=v \cdot v^{\prime}$ and $C_{c b}$ is a coefficient containing logarithmic corrections depending on $\alpha_{s}$; within our approximation it can be put equal to 1: $C_{c b}=1$. We also note that, in the leading order we are considering here $\xi(1)=1$. To compute $\xi$ one has to evaluate the diagram of Fig.2.

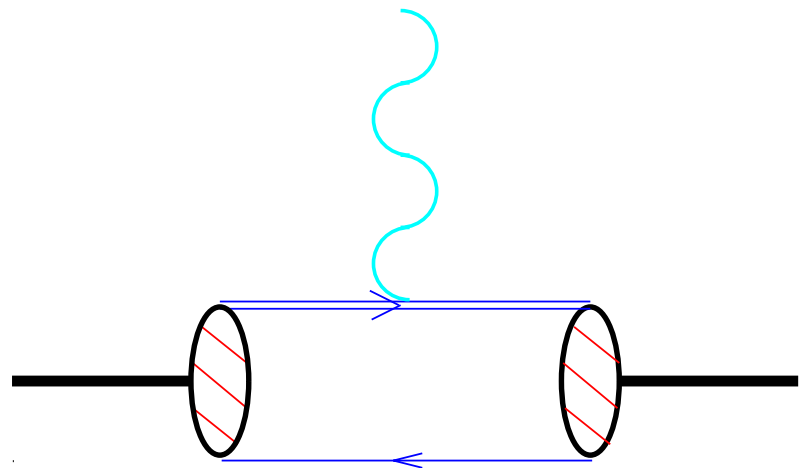

Fig. 2 - Weak current insertion on the heavy quark line for the heavy meson form factor calculation.

One finds [6]:

$$
\xi(\omega)=Z_{H}\left[\frac{2}{1+\omega} I_{3}\left(\Delta_{H}\right)+\left(m+\frac{2 \Delta_{H}}{1+\omega}\right) I_{5}\left(\Delta_{H}, \Delta_{H}, \omega\right)\right]
$$

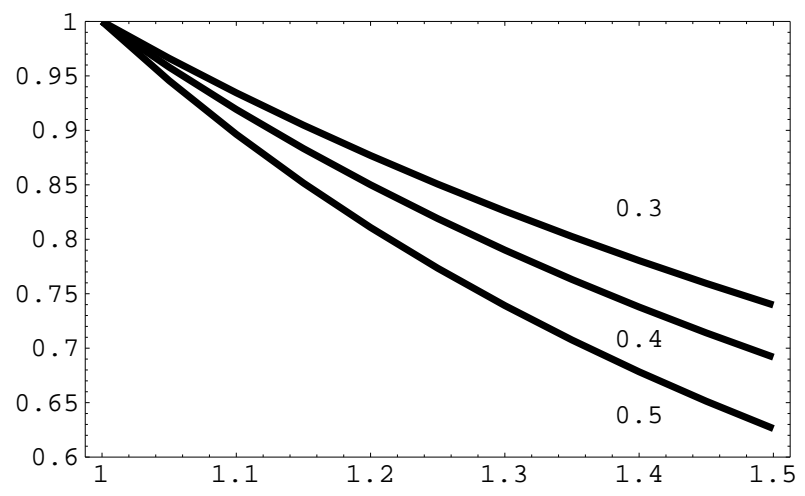

Fig. 3 - Isgur-Wise form factor at different $\Delta$ values. 
The $\xi$ function is plotted in Fig.3.

The integrals $I_{3}, I_{5}$ can be found in the Appendix. It is obvious from (32), (46) that $\xi(\omega)$ is correctly normalized, i.e. $\xi(1)=1$. For the slope of the Isgur-Wise function, defined by

$$
\rho_{I W}^{2}=-\frac{d \xi}{d \omega}(1)
$$

one gets the values reported in Table IV. The plot of the Isgur-Wise function is given in fig. 3 for three values of $\Delta_{H}$. It is useful to compare our results with other approaches. QCD sum rules calculations are in the range $\rho_{I W}^{2}=0.54-1.0$, therefore they agree with our results [18]. The results from different quark models [19], [20], [21], [22] have been recently reviewed by the authors of [23]; they have studied the results of different models with the common approach of boosting the wave-functions by the Bakamjian-Thomas method [24]; for $\rho_{I W}^{2}$ they obtain results in the range $0.97-1.28$, to be compared to the results in table 4 . Lattice QCD gives significantly smaller results; for example in [25] the value $\rho_{I W}^{2}(1)=0.64$ is obtained.

Let us now turn to the form factors describing the semileptonic decays of a meson belonging to the fundamental negative parity multiplet $H$ into the positive parity mesons in the $S$ and $T$ multiplets. Examples of these decays are

$$
B \rightarrow D^{* *} \ell \nu
$$

where $D^{* *}$ can be either a $S$ state (i.e. a $0^{+}$or $1^{+}$charmed meson having $s_{\ell}=1 / 2$ ) or a $T$ state ( i.e. a $2^{+}$or $1^{+}$ charmed meson having $s_{\ell}=3 / 2$ ).

The decays in (48) are described by two form factors $\tau_{1 / 2}, \tau_{3 / 2}$ [26] according to

$$
\begin{gathered}
<D_{2}^{*}\left(v^{\prime}, \epsilon\right)\left|\bar{c} \gamma_{\mu}\left(1-\gamma_{5}\right) b\right| B(v)>=\sqrt{3 M_{B} M_{D_{2}^{*}}} \tau_{3 / 2}(\omega) \times \\
{\left[i \epsilon_{\mu \alpha \beta \gamma} \epsilon^{* \alpha \eta} v_{\eta} v^{\beta} v^{\gamma}-\left[(\omega+1) \epsilon_{\mu \alpha}^{*} v^{\alpha}-\epsilon_{\alpha \beta}^{*} v^{\alpha} v^{\beta} v_{\mu}^{\prime}\right]\right]} \\
<D_{1}^{*}\left(v^{\prime}, \epsilon\right)\left|\bar{c} \gamma_{\mu}\left(1-\gamma_{5}\right) b\right| B(v)>=\sqrt{\frac{M_{B} M_{D_{1}^{*}}}{2}} \tau_{3 / 2}(\omega) \times \\
\left\{\left(\omega^{2}-1\right) \epsilon_{\mu}^{*}+\left(\epsilon^{*} \cdot v\right)\left[3 v_{\mu}-(\omega-2) v_{\mu}^{\prime}\right]-i(\omega+1) \epsilon_{\mu \alpha \beta \gamma} \epsilon^{* \alpha} v^{\prime \beta} v^{\gamma}\right\} \\
\left\langle D_{0}\left(v^{\prime}\right)\left|\bar{c} \gamma_{\mu}\left(1-\gamma_{5}\right) b\right| B(v)\right\rangle=\sqrt{M_{B} M_{D_{0}}} 2 \tau_{1 / 2}(\omega)\left(v_{\mu}^{\prime}-v_{\mu}\right) \\
\left\langle D_{1}^{* \prime}\left(v^{\prime}, \epsilon\right)\left|\bar{c} \gamma_{\mu}\left(1-\gamma_{5}\right) b\right| B(v)\right\rangle=\sqrt{M_{B} M_{D_{1}^{* \prime}}} \tau_{1 / 2}(\omega)\left\{2 i \epsilon_{\mu \alpha \beta \gamma} \epsilon^{* \alpha} v^{\prime \beta} v^{\gamma}\right. \\
\left.+2\left[(1-\omega) \epsilon_{\mu}^{*}+\left(\epsilon^{*} \cdot v\right) v_{\mu}^{\prime}\right]\right\} .
\end{gathered}
$$

In all these equations we neglect logarithmic corrections. The form factors $\tau_{1 / 2}(\omega), \tau_{3 / 2}(\omega)$ can be computed by a loop calculation similar to the one used to obtain $\xi(\omega)$. The result is

$$
\tau_{1 / 2}(\omega)=\frac{\sqrt{Z_{H} Z_{S}}}{2(1-\omega)}\left[I_{3}\left(\Delta_{S}\right)-I_{3}\left(\Delta_{H}\right)+\left(\Delta_{H}-\Delta_{S}+m(1-\omega)\right) I_{5}\left(\Delta_{H}, \Delta_{S}, \omega\right)\right]
$$

and

$$
\begin{aligned}
\tau_{3 / 2}(\omega) & =-\frac{\sqrt{Z_{H} Z_{T}}}{\sqrt{3}}\left[m \left(\frac{I_{3}\left(\Delta_{H}\right)-I_{3}\left(\Delta_{T}\right)-\left(\Delta_{H}-\Delta_{T}\right) I_{5}\left(\Delta_{H}, \Delta_{T}, \omega\right)}{2(1-\omega)}\right.\right. \\
& \left.-\frac{I_{3}\left(\Delta_{H}\right)+I_{3}\left(\Delta_{T}\right)+\left(\Delta_{H}+\Delta_{T}\right) I_{5}\left(\Delta_{H}, \Delta_{T}, \omega\right)}{2(1+\omega)}\right) \\
& -\frac{1}{2\left(-1-\omega+\omega^{2}+\omega^{3}\right)}\left(-3 S\left(\Delta_{H}, \Delta_{T}, \omega\right)-(1-2 \omega) S\left(\Delta_{T}, \Delta_{H}, \omega\right)\right. \\
& \left.\left.+\left(1-\omega^{2}\right) T\left(\Delta_{H}, \Delta_{T}, \omega\right)-2(1-2 \omega) U\left(\Delta_{H}, \Delta_{T}, \omega\right)\right)\right]
\end{aligned}
$$

where the integrals $S, T, U$ are defined in the appendix.

Given the small phase space which is available for these decays $\left(\omega_{\max }=1.33\right.$ for $D_{1}^{*}, D_{2}^{*}$ and $\omega_{\max } \simeq 1.215$ for $\left.D_{1}^{* \prime}, D_{0}\right)$, we can approximate 


$$
\tau_{j}(\omega) \simeq \tau_{j}(1) \times\left[1-\rho_{j}^{2}(\omega-1)\right] .
$$

Numerically we find the results reported in Table IV.

\begin{tabular}{lllllll}
\hline \hline$\Delta_{H}$ & $\xi(1)$ & $\rho_{I W}^{2}$ & $\tau_{1 / 2}(1)$ & $\rho_{1 / 2}^{2}$ & $\tau_{3 / 2}(1)$ & $\rho_{3 / 2}^{2}$ \\
\hline 0.3 & 1 & 0.72 & 0.08 & 0.8 & 0.48 & 1.4 \\
0.4 & 1 & 0.87 & 0.09 & 1.1 & 0.56 & 2.3 \\
0.5 & 1 & 1.14 & 0.09 & 2.7 & 0.67 & 3.0 \\
\hline \hline
\end{tabular}

TABLE IV. Form factors and slopes. $\Delta_{H}$ in GeV.

An important test of our approach is represented by the Bjorken sum rule, which states

$$
\rho_{I W}^{2}=\frac{1}{4}+\sum_{k}\left[\left|\tau_{1 / 2}^{(k)}(1)\right|^{2}+2\left|\tau_{3 / 2}^{(k)}(1)\right|^{2}\right] .
$$

Numerically we find that the first excited resonances, i.e. the $S$ and $T$ states $(k=0)$ practically saturate the sum rule for all the three values of $\Delta_{H}$.

In Table $\mathrm{V}$ we compare our results (for $\Delta_{H}=0.4 \mathrm{GeV}$ ) with other approaches. For $\tau_{3 / 2}$ we find a broad agreement with some of the constituent quark model results, whereas for $\tau_{1 / 2}$ we only agree with 34].

\begin{tabular}{lllll}
\hline \hline$\tau_{1 / 2}(1)$ & $\rho_{1 / 2}^{2}$ & $\tau_{3 / 2}(1)$ & $\rho_{3 / 2}^{2}$ & Ref. \\
\hline 0.09 & 1.1 & 0.56 & 2.3 & This work \\
0.41 & 1.0 & $0.41($ input $)$ & 1.5 & 27 \\
0.25 & 0.4 & 0.28 & 0.9 & 17 \\
0.31 & 2.8 & 0.31 & 2.8 & 22 \\
0.41 & 1.4 & 0.66 & 1.9 & 28, \\
0.059 & 0.73 & 0.515 & 1.45 & 23, \\
0.225 & 0.83 & 0.54 & 1.50 & 23 \\
\hline \hline
\end{tabular}

TABLE V. Parameters of the form factors $\tau_{1 / 2}, \tau_{3 / 2}$. The results in this table are for $\Delta_{H}=0.4 \mathrm{GeV}$.

Finally in Table VI we present our results for the branching ratios of B semileptonic decays to $S$ - and $P$-wave charmed mesons for three values of $\Delta_{H}$ computed with $V_{c b}=0.038$ [29] and $\tau_{B}=1.62$ psec. We see that data favor a value of $\Delta_{H} \simeq 400-500 \mathrm{MeV}$.

\begin{tabular}{lllll}
\hline \hline Decay mode & $\Delta_{H}=0.3$ & $\Delta_{H}=0.4$ & $\Delta_{H}=0.5$ & Exp. \\
\hline$B \rightarrow D \ell \nu$ & 3.0 & 2.7 & 2.2 & $1.9 \pm 0.5$ [14] \\
$B \rightarrow D^{*} \ell \nu$ & 7.6 & 6.9 & 5.9 & $4.68 \pm 0.25$ [14] \\
$B \rightarrow D_{0} \ell \nu$ & 0.03 & 0.005 & 0.003 & - \\
$B \rightarrow D_{1}^{* \prime} \ell \nu$ & 0.03 & 0.008 & 0.0045 & - \\
$B \rightarrow D_{1}^{*} \ell \nu$ & 0.27 & 0.18 & 0.13 & $0.74 \pm 0.16$ [3] \\
$B \rightarrow D_{2}^{*} \ell \nu$ & 0.43 & 0.34 & 0.30 & $<0.85$ \\
\hline
\end{tabular}

TABLE VI. Branching ratios (\%) for semileptonic $B$ decays. Theoretical predictions for three values of $\overline{\bar{~}}_{H}$ and experimental results (for $B^{0}$ decays). Units of $\Delta_{H}$ in $\mathrm{GeV}$. 


\section{STRONG AND RADIATIVE DECAYS OF HEAVY MESONS}

In this section we consider the strong decays

$$
\begin{aligned}
& H \rightarrow H \pi \\
& S \rightarrow H \pi
\end{aligned}
$$

as well as the radiative decay

$$
P^{*} \rightarrow P \gamma
$$

where $P^{*}$ and $P$ are the $1^{-}$and $0^{-}$members of the multiplet $H$.

\section{A. Strong decays}

The calculation of the strong coupling constants describing the decays $H \rightarrow H \pi$ (i.e. $D^{*} \rightarrow D \pi$ ) and $S \rightarrow H \pi$ is strongly simplified by adopting the soft pion limit. In the case of the decay $D^{*} \rightarrow D \pi$ this procedure introduces only a small error since the phase space is actually very small (for the $S \rightarrow H \pi$ decay the situation is different, see below).

Let us define $g_{D^{*} D \pi}$ by the equation:

$$
<\pi^{+}(q) D^{0}(p) \mid D^{*+}\left(p^{\prime}, \epsilon\right)>=i g_{D^{*} D \pi} \epsilon^{\mu} q_{\mu} .
$$

The constant $g_{D^{*} D \pi}$ is related to the strong coupling constant of the effective meson field theory $g$ appearing in [2]

$$
\mathcal{L}=i g \operatorname{Tr}\left(\bar{H} H \gamma^{\mu} \gamma_{5} \mathcal{A}_{\mu}\right)+\left[i h \operatorname{Tr}\left(\bar{H} S \gamma^{\mu} \gamma_{5} \mathcal{A}_{\mu}\right)+\text { h.c. }\right]
$$

by the relation

$$
g_{D * D \pi}=\frac{2 m_{D}}{f_{\pi}} g
$$

valid in the $m_{Q} \rightarrow \infty$ limit.

To compute $g$ in the soft-pion-limit $\left(q^{\mu} \rightarrow 0\right)$ we consider the matrix element of $\partial_{\mu} A^{\mu}$ and derive a GoldbergerTreiman relation, following the approach of [31]; this approach differs from the method employed in the first paper in [6], that assumes the so-called low-momentum-expansion approximation and a mixing between the Nambu-Goldstone bosons and higher mass resonances. Our method amounts to a loop calculation involving a current and two $H$ states (see Fig. 4).

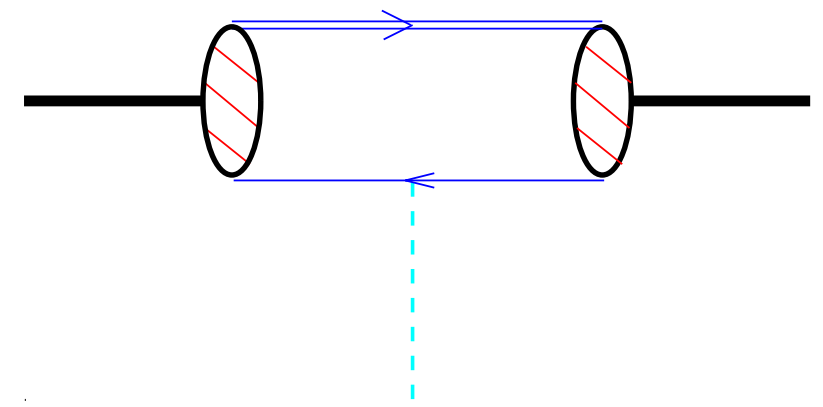

Fig. 4 - Pion vertex on the light quark line for the heavy-heavy-pion interaction.

The result for $g$ is as follows:

$$
g=Z_{H}\left[\frac{1}{3} I_{3}\left(\Delta_{H}\right)-2\left(m+\frac{1}{3} \Delta_{H}\right)\left(I_{2}+\Delta_{H} I_{4}\left(\Delta_{H}\right)\right)-\frac{4}{3} m^{2} I_{4}\left(\Delta_{H}\right)\right],
$$

where $I_{2}, I_{3}$ have been defined already and $I_{4}$ is in the Appendix. Numerically we get

$$
g=0.456 \pm 0.040
$$


where the central value corresponds to $\Delta_{H}=0.4 \mathrm{GeV}$ and the lower (resp. higher) value corresponds to $\Delta_{H}=0.3$ $\mathrm{GeV}$ (resp. $\Delta_{H}=0.5 \mathrm{GeV}$ ). These values agree with QCD sum rules calculations, that give $g=0.44 \pm 0.16$ (for a review see [2]; see also [32]), with the result of relativistic constituent quark model: $g \simeq 0.40$ [31], $g=0.34$ [33]. From the computed values of $g$ we can derive the hadronic width using

$$
\Gamma\left(D^{*+} \rightarrow D^{0} \pi^{+}\right)=\frac{g^{2}}{6 \pi f_{\pi}^{2}}\left|\vec{p}_{\pi}\right|^{3} .
$$

The numerical results will be discussed at the end of this Section. Let us now comment on the strong decay $S \rightarrow H \pi$. A complete calculation of the corresponding decay constant $h$ appearing in (61) is much more involved and one can preliminary try to compute it in the soft-pion limit. This approximation implies that we must assume $\Delta_{S}=\Delta_{H}$; putting $\Delta=\left(\Delta_{H}+\Delta_{S}\right) / 2$, we obtain

$$
h=\bar{Z}\left\{I_{3}(\Delta)+2 \Delta I_{2}+2\left(\Delta^{2}-m^{2}\right) I_{4}(\Delta)\right\}
$$

were $\bar{Z}$ is given by

$$
\bar{Z}=\left[\left(I_{3}(\Delta)+\Delta \frac{\partial I_{3}}{\partial \Delta}\right)^{2}-\left(m \frac{\partial I_{3}}{\partial \Delta}\right)^{2}\right]^{-\frac{1}{2}} .
$$

We obtain, for $\Delta$ in the range $0.43-0.57 \mathrm{GeV}$ the result

$$
h=-0.85 \pm 0.02
$$

which is somehow higher, but still compatible, within the theoretical uncertainties, with a result obtained by QCD sum rules: $h=-0.52 \pm 0.17$ 15].

\section{B. Radiative decays}

Let us now consider the radiative decays $D^{*} \rightarrow D \gamma$ and $B^{*} \rightarrow B \gamma$. To be definite we consider only the former decay; moreover we use the SU(3) flavor symmetry for the light quarks. The matrix element for this radiative transition is the following:

$$
\mathcal{M}\left(D^{*} \rightarrow D \gamma\right)=i e \mu \epsilon^{\mu \nu \alpha \beta} \epsilon_{\mu}^{*} \eta_{\nu} p_{\alpha}^{\prime} p_{\beta}
$$

where $\epsilon_{\mu}$ is the photon polarization and the coupling $\mu$ comprises two terms:

$$
\mu=\mu^{\ell}+\mu^{h}
$$

corresponding to the decomposition:

$$
\begin{aligned}
\mathcal{M}\left(D^{*}\right. & \rightarrow D \gamma)=e \epsilon^{* \mu}<D\left(p^{\prime}\right)\left|J_{\mu}^{e m}\right| D^{*}(p, \eta)> \\
& =e \epsilon^{* \mu}<D\left(p^{\prime}\right)\left|J_{\mu}^{\ell}+J_{\mu}^{h}\right| D^{*}(p, \eta)>.
\end{aligned}
$$

Here $J_{\mu}^{\ell}$ and $J_{\mu}^{h}$ are the light and the heavy quark parts of the electro-magnetic current:

$$
J_{\mu}^{\ell}=\frac{2}{3} \bar{u} \gamma_{\mu} u-\frac{1}{3} \bar{d} \gamma_{\mu} d-\frac{1}{3} \bar{s} \gamma_{\mu} s
$$

and

$$
J_{\mu}^{h}=\frac{2}{3} \bar{c} \gamma_{\mu} c-\frac{1}{3} \bar{b} \gamma_{\mu} b=\sum_{Q=c, b} e_{Q} \bar{Q} \gamma_{\mu} Q
$$

Correspondingly, eq. (70) becomes

$$
\mu=\mu^{\ell}+\mu^{h}=\frac{e_{q}}{\Lambda_{q}}+\frac{e_{Q}}{\Lambda_{Q}},
$$


where $\Lambda_{q}$ and $\Lambda_{Q}$ are mass parameters to be determined. The photon insertion on the heavy quark line generates the $\mu^{h}$ coupling, while the $\mu^{\ell}$ arises when the photon is inserted on the light quark line of the loop.

In the $m_{Q} \rightarrow \infty$ limit, the matrix element of $J_{\mu}^{h}$ can be expressed in terms of the Isgur-Wise form factor $\xi(\omega)$ as follows:

$$
\begin{gathered}
<D\left(p^{\prime}\right)\left|J_{\mu}^{h}\right| D^{*}(p, \eta)>=e_{c}<D\left(p^{\prime}\right)\left|\bar{c} \gamma^{\mu} c\right| D^{*}(p, \eta)> \\
=i \frac{2}{3} \sqrt{M_{D} M_{D^{*}}} \xi(\omega) \epsilon_{\mu \nu \alpha \beta} \eta^{\nu} v^{\prime \alpha} v^{\beta}
\end{gathered}
$$

where $p^{\prime}=M_{D} v^{\prime}, p=M_{D^{*}} v$ and $\omega \simeq 1$ because:

$$
0=q^{2}=m_{D}^{2}+M_{D^{*}}^{2}-2 M_{D} M_{D^{*}} v \cdot v^{\prime}
$$

Taking into account the normalization $\xi(1)=1$ one gets, for the charm and beauty mesons respectively (with $\bar{q}=\bar{d})$

$$
\mu^{h}=\frac{2}{3 \Lambda_{c}} \quad \mu^{h}=-\frac{1}{3 \Lambda_{b}}
$$

with

$$
\Lambda_{c}=\sqrt{M_{D} M_{D^{*}}} \quad \Lambda_{b}=\sqrt{M_{B} M_{B^{*}}} .
$$

In the leading order in $1 / m_{c}$ and $1 / m_{b}$ one finds:

$$
\Lambda_{c}=m_{c} \quad \Lambda_{b}=m_{b}
$$

which fixes $\mu^{h}$. As for the coupling $\mu^{\ell}$, in our approach it stems from the diagram where the photon line is inserted on the light quark propagator (see Fig. 5).

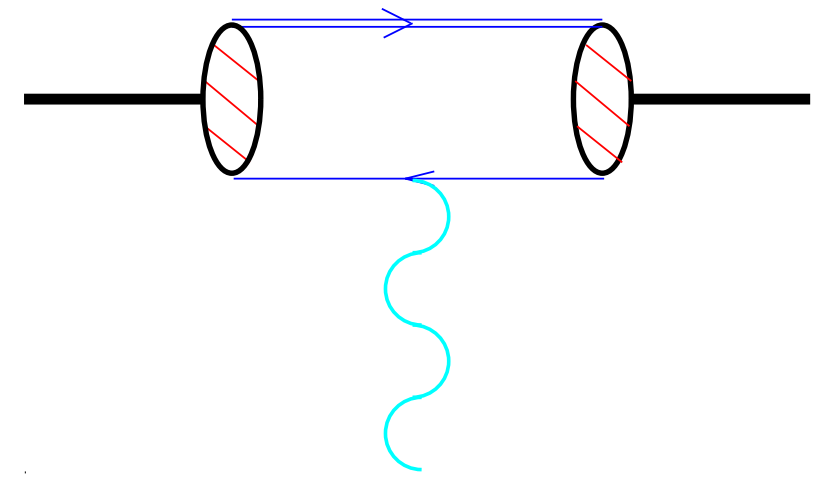

Fig. 5 - Electro-magnetic current insertion on the light quark line.

The result is:

$$
\beta=\Lambda_{q}^{-1}=2 \times Z_{H}\left[I_{2}+\left(m+\Delta_{H}\right) I_{4}\left(\Delta_{H}\right)\right]
$$

Numerically we get:

$$
\beta=1.6 \pm 0.1 \mathrm{GeV}^{-1}
$$

where the central value corresponds to $\Delta_{H}=0.4 \mathrm{GeV}$ and the lower (resp. higher) value corresponds to $\Delta_{H}=0.5$ $\mathrm{GeV}$ (resp. $\Delta_{H}=0.3 \mathrm{GeV}$ ). These values agree with the result of Heavy Quark Effective Theory and the Vector Meson Dominance hypothesis [34].

One can use the formula:

$$
\Gamma\left(D^{*} \rightarrow D \gamma\right)=\frac{\alpha}{3} \frac{M_{D^{*}}}{M_{D}}|\mu|^{2}|\vec{k}|^{3}
$$


$(\vec{k}=$ photon momentum $)$, to compute the $D^{*}$ radiative widths. Using this equation together with $(65)$ we obtain the results of Table VII.

\begin{tabular}{llll}
\hline \hline Decay mode & $\Delta_{H}=0.4 \mathrm{GeV}$ & $\Delta_{H}=0.5 \mathrm{GeV}$ & Exp. \\
\hline$D^{* 0} \rightarrow D^{0} \pi^{0}$ & 65.5 & 70.1 & $61.9 \pm 2.9$ \\
$D^{* 0} \rightarrow D^{0} \gamma$ & 34.5 & 29.9 & $38.1 \pm 2.9$ \\
$D^{*+} \rightarrow D^{0} \pi^{+}$ & 71.6 & 71.7 & $68.3 \pm 1.4$ \\
$D^{*+} \rightarrow D^{+} \pi^{0}$ & 28.0 & 28.1 & $30.6 \pm 2.5$ \\
$D^{*+} \rightarrow D^{+} \gamma$ & 0.4 & 0.24 & $1.1_{-0.7}^{+2.1}$ \\
\hline \hline
\end{tabular}

TABLE VII. Theoretical and experimental $D^{*}$ branching ratios (\%). Theoretical values are computed with $\Delta_{H}=0.4,0.5$ $\mathrm{GeV}$.

Taking into account the approximations involved in the present calculation, we find comparison between theoretical prediction and experimental data of Table VII encouraging. Finally we compute total widths for $D^{*}$ and $B^{*}$ (for $\left.\Delta_{H}=0.4 \mathrm{GeV}\right)$ :

$$
\begin{aligned}
\Gamma\left(D^{* 0}\right) & =38 \mathrm{KeV} \\
\Gamma\left(D^{*+}\right) & =62 \mathrm{KeV} \\
\Gamma\left(B^{* 0} \rightarrow B^{0} \gamma\right) & =0.05 \mathrm{KeV}
\end{aligned}
$$

\section{CONCLUSIONS}

The increasing number of available data on heavy meson processes and the even more promising increase of data from forthcoming experiments demands theoretical predictions for these processes to be compared with experiment and to suggest subsequent lines of investigation. Calculating directly from the QCD lagrangian remains an extremely difficult task, in spite of the impressive success of recent lattice work. A most promising approach is the one based on heavy meson effective lagrangians, which incorporate the heavy quark symmetries and in addition the approximate chiral symmetry for light quarks. Although with increasing data such an approach will remain the best one beyond direct QCD calculations, at this stage it is made cumbersome by the large number of parameters that have to be fixed before obtaining predictions. In this note we have presented an intermediate approach, not as rigorous and general as that of the effective meson lagrangian, but which allows for a smaller number of input parameters. We start from an effective lagrangian at the level of mesons and of constituent quarks, and we then calculate the meson transition amplitudes by evaluating loops of heavy and light quarks. In this way we can compute the Isgur-Wise function, the form factors $\tau_{1 / 2}$ and $\tau_{3 / 2}$, the leptonic decay constant $\hat{F}$ and $\hat{F}^{+}$, the coupling constants $g$ and $h$ relative to $H \rightarrow H \pi$ and $H \rightarrow S \pi$ processes respectively, the $\beta$ coupling relative to the $H \rightarrow H \gamma$ processes. The agreement with data, when available, seems rather impressive. Additional data should be able to confirm some of the predictions of the model or suggest modifications. In conclusion the model presented here, based on meson and quark degrees of freedom, seems capable of incorporating a number of essential features of QCD and to provide a useful approach to calculate heavy meson transitions in terms of a limited number of physical parameters. 


\section{APPENDIX}

We list here the integrals used in the paper.

$$
\begin{aligned}
I_{0}(\Delta) & =\frac{i N_{c}}{16 \pi^{4}} \int^{\mathrm{reg}} \frac{d^{4} k}{(v \cdot k+\Delta+i \epsilon)} \\
& =\frac{N_{c}}{16 \pi^{3 / 2}} \int_{1 / \Lambda^{2}}^{1 / \mu^{2}} \frac{d s}{s^{3 / 2}} e^{-s\left(m^{2}-\Delta^{2}\right)}\left(\frac{3}{2 s}+m^{2}-\Delta^{2}\right)[1+\operatorname{erf}(\Delta \sqrt{s})] \\
& -\Delta \frac{N_{c} m^{2}}{16 \pi^{2}} \Gamma\left(-1, \frac{m^{2}}{\Lambda^{2}}, \frac{m^{2}}{\mu^{2}}\right) \\
I_{1} & =\frac{i N_{c}}{16 \pi^{4}} \int^{r e g} \frac{d^{4} k}{\left(k^{2}-m^{2}\right)}=\frac{N_{c} m^{2}}{16 \pi^{2}} \Gamma\left(-1, \frac{m^{2}}{\Lambda^{2}}, \frac{m^{2}}{\mu^{2}}\right) \\
I_{1}^{\prime} & =\frac{i N_{c}}{16 \pi^{4}} \int^{\mathrm{reg}} d^{4} k \frac{k^{2}}{\left(k^{2}-m^{2}\right)}=\frac{N_{c} m^{4}}{8 \pi^{2}} \Gamma\left(-2, \frac{m^{2}}{\Lambda^{2}}, \frac{m^{2}}{\mu^{2}}\right) \\
I_{2} & =-\frac{i N_{c}}{16 \pi^{4}} \int^{\mathrm{reg}} \frac{d^{4} k}{\left(k^{2}-m^{2}\right)^{2}}=\frac{N_{c}}{16 \pi^{2}} \Gamma\left(0, \frac{m^{2}}{\Lambda^{2}}, \frac{m^{2}}{\mu^{2}}\right) \\
I_{3}(\Delta) & =-\frac{i N_{c}}{16 \pi^{4}} \int^{\mathrm{reg}} \frac{d^{4} k}{\left(k^{2}-m^{2}\right)(v \cdot k+\Delta+i \epsilon)} \\
& =\frac{N_{c}}{16 \pi^{3 / 2}} \int_{1 / \Lambda^{2}}^{1 / \mu^{2}} \frac{d s}{s^{3 / 2}} e^{-s\left(m^{2}-\Delta^{2}\right)}(1+\operatorname{erf}(\Delta \sqrt{s})) \\
I_{4}(\Delta) & =\frac{i N_{c}}{16 \pi^{4}} \int^{\mathrm{reg}} \frac{d^{4} k}{\left(k^{2}-m^{2}\right)^{2}(v \cdot k+\Delta+i \epsilon)} \\
& =\frac{N_{c}}{16 \pi^{3 / 2}} \int_{1 / \Lambda^{2}}^{1 / \mu^{2}} \frac{d s}{s^{1 / 2}} e^{-s\left(m^{2}-\Delta^{2}\right)}[1+\operatorname{erf}(\Delta \sqrt{s})] .
\end{aligned}
$$

In these equations

$$
\Gamma\left(\alpha, x_{0}, x_{1}\right)=\int_{x_{0}}^{x_{1}} d t e^{-t} t^{\alpha-1}
$$

is the generalized incomplete gamma function and erf is the error function.

Let's introduce now:

$$
\sigma\left(x, \Delta_{1}, \Delta_{2}, \omega\right)=\frac{\Delta_{1}(1-x)+\Delta_{2} x}{\sqrt{1+2(\omega-1) x+2(1-\omega) x^{2}}} .
$$

Then the integrals used for the semileptonic decays are as follows:

$$
\begin{aligned}
& I_{5}\left(\Delta_{1}, \Delta_{2}, \omega\right)= \frac{i N_{c}}{16 \pi^{4}} \int^{\mathrm{reg}} \frac{d^{4} k}{\left(k^{2}-m^{2}\right)\left(v \cdot k+\Delta_{1}+i \epsilon\right)\left(v^{\prime} \cdot k+\Delta_{2}+i \epsilon\right)} \\
&= \int_{0}^{1} d x \frac{1}{1+2 x^{2}(1-\omega)+2 x(\omega-1)} \times \\
& {\left[\frac{6}{16 \pi^{3 / 2}} \int_{1 / \Lambda^{2}}^{1 / \mu^{2}} d s \sigma e^{-s\left(m^{2}-\sigma^{2}\right)} s^{-1 / 2}(1+\operatorname{erf}(\sigma \sqrt{s}))+\right.} \\
&\left.\frac{6}{16 \pi^{2}} \int_{1 / \Lambda^{2}}^{1 / \mu^{2}} d s e^{-s\left(m^{2}-2 \sigma^{2}\right)} s^{-1}\right] \\
& I_{6}\left(\Delta_{1}, \Delta_{2}, \omega\right)= \frac{i N_{c}}{16 \pi^{4}} \int^{\mathrm{reg}} \frac{\frac{d^{4} k}{\left(v \cdot k+\Delta_{1}+i \epsilon\right)\left(v^{\prime} \cdot k+\Delta_{2}+i \epsilon\right)}}{=} \\
& I_{1} \int_{0}^{1} d x \frac{\sigma}{1+2 x^{2}(1-\omega)+2 x(\omega-1)}
\end{aligned}
$$




$$
\begin{aligned}
- & \frac{N_{c}}{16 \pi^{3 / 2}} \int_{0}^{1} d x \frac{1}{1+2 x^{2}(1-\omega)+2 x(\omega-1)} \times \\
& \int_{1 / \Lambda^{2}}^{1 / \mu^{2}} \frac{d s}{s^{3 / 2}} e^{-s\left(m^{2}-\sigma^{2}\right)}\left\{\sigma[1+\operatorname{erf}(\sigma \sqrt{s})] \cdot\left[1+2 s\left(m^{2}-\sigma^{2}\right)\right]\right. \\
+ & 2 \sqrt{\frac{s}{\pi}} e^{-s \sigma^{2}}\left[\frac{3}{2 s}+\left(m^{2}-\sigma^{2}\right)\right]
\end{aligned}
$$

$$
\begin{aligned}
& S\left(\Delta_{1}, \Delta_{2}, \omega\right)=\Delta_{1} I_{3}\left(\Delta_{2}\right)+\omega\left(I_{1}+\Delta_{2} I_{3}\left(\Delta_{2}\right)\right)+\Delta_{1}^{2} I_{5}\left(\Delta_{1}, \Delta_{2}, \omega\right) \\
& T\left(\Delta_{1}, \Delta_{2}, \omega\right)=m^{2} I_{5}\left(\Delta_{1}, \Delta_{2}, \omega\right)+I_{6}\left(\Delta_{1}, \Delta_{2}, \omega\right) \\
& U\left(\Delta_{1}, \Delta_{2}, \omega\right)=I_{1}+\Delta_{2} I_{3}\left(\Delta_{2}\right)+\Delta_{1} I_{3}\left(\Delta_{1}\right)+\Delta_{2} \Delta_{1} I_{5}\left(\Delta_{1}, \Delta_{2}, \omega\right)
\end{aligned}
$$




\section{Acknowledgments}

A.D. acknowledges the support of a "Marie Curie" TMR research fellowship of the European Commission under contract ERBFMBICT960965. He also thanks L. Lellouch for a discussion on lattice data and $\mathrm{O}$. Pène for discussion on ref. 23]. This work has also been carried out in part under the EC program Human Capital and Mobility, contract UE ERBCHRXCT940579 and OFES 950200 .

[1] For further references see the review papers: H. Georgi, contribution to the Proceedings of TASI 91, R.K. Ellis ed., World Scientific, Singapore,1991; B. Grinstein, contribution to High Energy Phenomenology, R. Huerta and M.A. Peres eds., World Scientific, Singapore, 1991; N. Isgur and M. Wise, contribution to Heavy Flavours, A. Buras and M. Lindner eds., World Scientific, Singapore,1992; M. Neubert, Phys. Rep. 245259 (1994).

[2] R. Casalbuoni, A. Deandrea, N. Di Bartolomeo, R. Gatto, F. Feruglio, G. Nardulli, Phys. Rep. 281145 (1997).

[3] See for example: J. M. Flynn, C. T. Sachrajda, heplat/9710080 and references therein.

[4] A. Manohar and H. Georgi, Nucl. Phys. B234 189 (1984).

[5] J. Bijnens, Phys. Rep. 265369 (1996); T. Hatsuda and T. Kunihiro, Phys. Rep. 247221 (1994).

[6] D. Ebert, T. Feldmann, R. Friedrich and H. Reinhardt, Nucl. Phys. B434 619 (1995); D. Ebert, T. Feldmann and H. Reinhardt, Phys. Lett. B388 154 (1996).

[7] D. Buskulic et al., ALEPH Collaboration, Z. Phys. C73 60 (1997); ibidem, Phys. Lett. B395 373 (1997); K. Ackerstaff et al., OPAL Collaboration, Z.Phys. C76 425 (1997); P. Avery et al., CLEO Collaboration, Phys. Lett. B331 236 (1994), Erratum, ibidem B342 453 (1994).

[8] J. D. Bjorken, talk at Les Rencontres de la Vallée d'Aoste, La Thuile, Italy, SLAC Report SLAC-PUB5278, 1990 (unpublished); SLAC 1990 Summer Institute, SLAC Report-378, pp. 167-198.

[9] T. Mannel, W. Roberts and Z. Ryzak, Nucl. Phys. B368 204 (1992).

[10] J. M. Flynn and N. Isgur, J. Phys. G18 1627 (1992).

[11] A. Falk and M. Luke Phys. Lett. B292 119 (1992).

[12] B. Holdom and M. Sutherland, Phys. Rev. D47 5067 (1993), B. Holdom and M. Sutherland, Phys. Lett. B313 (1993) 447, B. Holdom and M. Sutherland, Phys. Rev. D48 (1993) 5196, B. Holdom, M. Sutherland and J. Mureika, Phys. Rev. D49 (1994) 2359.

[13] W. H. Bardeen and C. T. Hill, Phys. Rev. D49 409 (1994).

[14] R.M. Barnett et al. (Particle Data Group), Phys. Rev. D54 1 (1996) and 1997 off-year partial update for the
1998 edition, http://pdg.lbl.gov

[15] P. Colangelo, F. De Fazio, G. Nardulli, N. Di Bartolomeo and R. Gatto, Phys. Rev. D 526422 (1995).

[16] M. Neubert, Phys. Rev. D45 2451 (1992).

[17] P. Colangelo, G. Nardulli and N. Paver, Phys. Lett. B293 207 (1992).

[18] B. Blok and M. Shifman, Phys. Rev. D47 2949 (1993); M. Neubert, Phys. Rep. 245259 (1994); E. Bagan, P. Ball and P. Gosdzinsky, Phys. Lett. B301 249 (1993); S. Narison, report CERN-TH 7103/93, PM-93/45.

[19] S. Godfrey and N. Isgur, Phys. Rev. D32 189 (1985).

[20] S. Veseli and I. Dunietz, Phys. Rev. D54 6803 (1996).

[21] P. Cea, P. Colangelo, L. Cosmai and G. Nardulli, Phys. Lett. B 206691 (1988); P. Colangelo, G. Nardulli and M. Pietroni, Phys. Rev. D43 3002 (1991).

[22] N. Isgur, D. Scora, B. Grinstein and M.B. Wise D39 799 (1989).

[23] V. Morenas, A. Le Yaouanc, L. Oliver, O. Pène and J.-C. Raynal, Phys. Rev. D56 5668 (1997).

[24] B. Bakamjian and L.H. Thomas, Phys. Rev. 921300 (1953).

[25] J. Christensen, T. Draper and C. McNeile, heplat/9710025.

[26] N. Isgur and M. B. Wise, Phys. Rev. D43 819 (1991).

[27] A. K. Leibovich, Z. Ligeti, I. W. Stewart and M. B. Wise, Phys. Rev. Lett. 663995 (1997); hep-ph/9705467.

[28] A. Wambach, Nucl. Phys. B434 647 (1995).

[29] M. Neubert, hep-ph/9801269, in International Europhysics Conference on High Energy Physics, Jerusalem, Israel, 19-26 August 1997.

[30] See the first two references in [7].

[31] P. Colangelo, F. De Fazio and G. Nardulli, Phys. Lett. B334 175 (1994).

[32] A. A. Ovchinnikov, Sov. J. of Nucl. Phys. 50519 (1989); P. Colangelo, G. Nardulli, A. Deandrea, N. Di Bartolomeo, R. Gatto and F. Feruglio, Phys. Lett. B339 151 (1994); V.M. Belyaev, V.M. Braun, A. Khodzamirian, R. Rueckl, Phys. Rev. D51 6177 (1995); T.M. Aliev, D.A. Demir, E. Iltan and N.K. Pak, Phys. Lett. B351 339 (1995).

[33] M. Sutherland, B. Holdom, S. Jaimungal, and R. Lewis, Phys. Rev. D51 (1995) 5053.

[34] P. Colangelo, F. De Fazio and G. Nardulli, Phys. Lett. B316 555 (1993). 